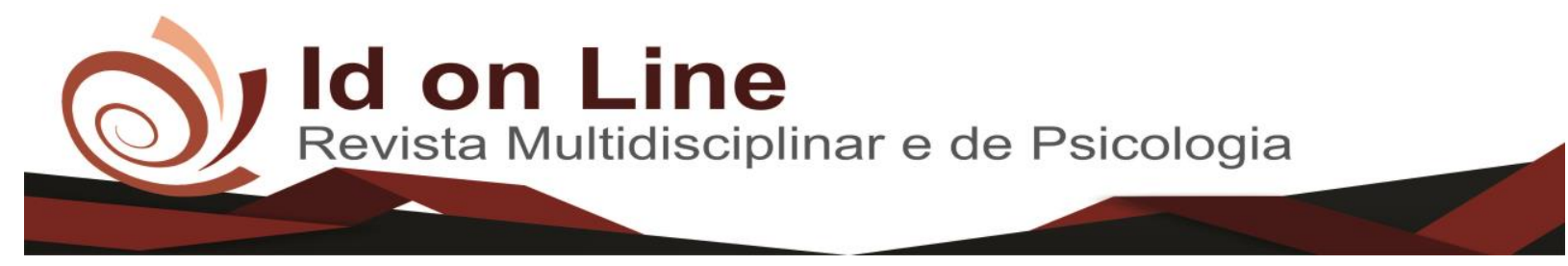

Artigo de Revisão

\title{
A Relação entre Depressão e Ideação Suicida na Juventude
}

\author{
Abigail Virginia Miranda de Alencar ${ }^{1}$; Thércia Lucena Grangeiro Maranhão ; \\ Rivalina Maria Macedo Fernandes ${ }^{3}$; Maria do Socorro Rodrigues ${ }^{4}$
}

\begin{abstract}
Resumo: O presente estudo tem como objetivo geral: identificar a relação entre depressão e ideação suicida por meio da literatura existente. Tendo como objetivos específicos: analisar as publicações mais recentes acerca de depressão e ideação suicida na juventude; compreender como ocorre a relação entre a depressão e a ideação suicida em jovens; investigar quais as intervenções clínicas da psicologia em casos de depressão e ideação suicida na juventude. A escolha do tema justifica-se pelo fato dos pesquisadores estarem vinculados a grande área da ciências da saúde, havendo, portanto, a necessidade de aprofundar seus conhecimentos, uma vez que os casos de depressão com ideação suicida apresentam importante incidência na juventude, o que torna imprescindível a existência de reflexões efetivas na área as saúde e psicologia. Para o alcance dos objetivos previamente estabelecidos no estudo, foi realizada uma revisão de literatura acerca do tema proposto. A pesquisa traz contribuições no que concerne ao conhecimento a respeito da referida temática, identificando subsídios teóricos que auxiliarão no desenvolvimento de outros estudos acerca da depressão e ideação suicida na juventude , refletindo sobre as intervenções clinicas, auxiliando na prática de profissionais que atuam na referida área.
\end{abstract}

Palavras-chave: Depressão. Ideação Suicida. Juventude

\section{The Relationship Between Depression and Suicidal Idea in Youth}

\begin{abstract}
The present study aims to identify the relationship between depression and suicidal ideation through existing literature. With specific objectives: to analyze the most recent publications about depression and suicidal ideation in youth; understand how the relationship between depression and suicidal ideation in young people occurs; to investigate the clinical interventions of psychology in cases of depression and suicidal ideation in youth. The choice of theme is justified by the fact that researchers are linked to a large area of health sciences, and there is a need to deepen their knowledge, since cases of depression with suicidal ideation have an important impact on youth, which makes it essential to have effective reflections in the area of health and psychology. To reach the objectives previously established in the study, a literature review was carried out on the proposed theme The research brings contributions regarding the knowledge about this subject, identifying theoretical subsidies that will help in the development of other studies about depression and suicidal ideation in youth, reflecting on the clinical interventions, assisting in the practice of professionals who work in that area.
\end{abstract}

Keywords: Depression. Suicidal Ideas. Youth

\footnotetext{
${ }^{1}$ Acadêmica do Curso de Graduação em Psicologia do Centro Universitário Doutor Leão Sampaio. E-mail: abigail_alencar@hotmail.com ${ }^{2}$ Psicóloga. Professora do Curso de Graduação em Psicologia e Gestão de RH do Centro Universitário Doutor Leão Sampaio- UNILEÃO. Mestre em Ciências da Saúde pela FMABC.E-mail: thercia@leaosampaio.edu.br;

${ }^{3}$ Mestra em Gestão em Organizações Aprendentes (UFPB), Pós - Graduação Lato Sensu Especialização em Psicopedagogia (Universidade Estadual Vale do Acaraú - UVA), Pós - Graduação Lato Sensu Especialização em Educação Especial (Faculdade Internacional de Curitiba FACINTER), Pós - Graduação Lato Sensu Especialização em Gestão de Pessoas (UFPB), Graduação em Psicologia Clinica e organizacional (UNIPÊ), Licenciatura em Psicologia (UNIPÊ). Docente em Cursos de Pós - Graduação Lato Sensu (UVA) e graduação na Faculdade Integrada de Cruzeiro (FIC). Email: rivalinafernandes@ gmail.com;

${ }^{4}$ Graduada em Psicologia pelo Centro Universitário Doutor Leão Sampaio. Contato: socorro.rodrigues@yahoo.com.br.
} 


\section{Introdução}

O número de indivíduos com transtorno depressivo teve um aumento de $18 \%$ entre os anos de 2005 e 2015,atualmente há em média 322 milhões de pessoas com depressão no mundo. No Brasil, esse transtorno chega a atingir 5,8 da população. Constituindo-se como a principal causa de incapacidade no mundo inteiro, a depressão tem uma importante colaboração na carga global de doenças (OMS, 2017).

A depressão é considerada como a alteração afetiva mais discutida e estudada nos dias atuais. Conceituada como um transtorno de humor, a depressão vem controlar as atitudes dos indivíduos alterando a forma como esses sujeitos percebem a si mesmos, levando-os a observarem seus problemas como sérias catástrofes. O transtorno depressivo é caracterizado por alguns sintomas como irritabilidade, apatia, tristeza, perda de interesse, retardo motor ou agitação, pensamentos agressivos, angústia e diversas queixas somáticas como:fadiga, insônia, anorexia. (ESTEVES; GALVAN, 2006).

No pior dos casos, a depressão pode levar o individuo a cometer suicídio. Estima-se que aproximadamente 800 mil pessoas cometem suicídio anualmente, sendo considerada como a segunda principal causa de morte de indivíduos na faixa etária dos 15 a 29 anos (OPAS, 2016). De acordo com Moura; Bastos (2015), as estatísticas acerca do suicídio são falhas e subestimadas, especialmente no que se refere aos adolescentes, uma vez que suas ações autodestrutivas, geralmente são omitidas e escondidas pela família.

A ideação suicida apresenta-se como um dos mais importantes precedentes do suicídio. Caracteriza-se por ideias pensamentos sobre o suicídio e/ou planejamento da própria morte. Abrange anseios, atitudes, condutas ou planos que o sujeito possua para se matar. O fato de haver ideias suicidas constitui um alto risco para a execução de uma tentativa de suicídio, existindo do ponto de vista empírico uma forte associação entre as duas variáveis (LARANJEIRA, 2015).

Nesse sentido, a juventude, por tratar-se de uma fase de desenvolvimento permeado por várias transformações de ordem biológica, psicológica e social que, na maioria das vezes, são seguidas de conflitos e angustias, tem-se percebido nos últimos anos, um aumento no comportamento suicida entre adolescentes. O suicídio na juventude é pouco estudado, já que apresenta etiologia complexa e abrange diferentes contextos da vida do jovem (MOREIRA; BASTOS, 2015). 
No que se refere aos cuidados psicológicos, sabe-se que estes são indispensáveis no caso do suicídio. As tentativas de suicídio não devem ser desvalorizadas e nem supervalorizadas, faz-se necessário compreendê-las e acolhê-las forma eficiente (ZANA; KOVÁCS, 2013).

Desse modo, o presente estudo tem como objetivo geral: identificar a relação entre depressão e ideação suicida por meio da literatura existente. Tendo como objetivos específicos: analisar as publicações mais recentes acerca de depressão e ideação suicida na juventude; compreender como ocorre a relação entre a depressão e a ideação suicida em jovens; investigar quais as intervenções clínicas da psicologia em casos de depressão e ideação suicida na juventude.

A escolha do tema justifica-se pelo fato dos pesquisadores estarem vinculados a grande área da ciências da saúde, havendo, portanto, a necessidade de aprofundar seus conhecimentos, uma vez que os casos de depressão com ideação suicida apresentam importante incidência na juventude, o que torna imprescindível a existência de intervenções efetivas da medicina e psicologia.

O trabalho traz contribuições no que concerne ao conhecimento a respeito da referida temática, identificando subsídios teóricos que auxiliarão no desenvolvimento de outros estudos acerca da depressão e ideação suicida na juventude , refletindo sobre as intervenções clinicas ,auxiliando na prática de profissionais que atuam na referida área.

Para o alcance dos objetivos previamente estabelecidos no estudo, foi realizada uma revisão de literatura acerca do tema proposto.

\section{Metodologia}

O método adotado foi a revisão de literatura sobre a relação entre depressão e ideação suicida na juventude que apontavam para possíveis intervenções clínicas. De acordo com Andrade et al. (2016), esse tipo de pesquisa possui caráter bibliográfico e é realizada através da revisão de resultados de estudos, com o objetivo de planejar articulações para estabelecer novos conhecimentos. 
As informações disponíveis na literatura acerca da relação entre depressão e ideação suicida na juventude e as intervenções clínicas da psicologia, foram colhidas a partir das bases de dados SciELO, BVS psicologia Brasil e LILACS.

$\mathrm{Na}$ busca dos estudos e pesquisas foram utilizados os seguintes descritores: “Depressão e Suicídio “, Juventude ”, "Ideação Suicida”, “Ideação Suicida em Adolescentes”.

Para a seleção dos artigos, foram obedecidos os seguintes critérios de inclusão: terem sido publicados no período compreendido entre os anos de 2011 e 2017; está disponível no idioma português; ter em seu título e/ou resumo, pelo menos uma das palavras chave. Foram estabelecidos como critérios de exclusão: artigos publicados em data anterior a 2011; artigos que não possuam nenhuma das palavras chave.

Após a pesquisa, seleção e leitura dos artigos, foi realizado um fichamento dos dados obtidos com o objetivo de sintetizar e auxiliar para melhor compreensão das informações. Para a análise dos dados foi utilizada a abordagem qualitativa. Serão apresentadas considerações acerca da juventude; depressão; ideação suicida e; suicídio.

\section{Considerações Sobre a Juventude}

O conceito de juventude é um tema abrangente e tem uma maior integração de assuntos sociais, históricos, econômicos, culturais, políticos, territoriais, dentre outros aspectos. Dessa forma, seus conceitos dependem de transformações históricas. A ideia de juventude emerge associada a um processo temporal que expressa movimentos humanos rumo a um objetivo de idealização, nesse caso a maturidade do intelecto. É percebida como uma faixa etária, compreendida entre os 15 e 24 anos (MOREIRA; ROSÁRIO; SANTOS, 2011).

Existem muitas concepções para definir o que é a juventude, nas quais se utilizam diferentes critérios para se aproximar mais daquilo que são os jovens. Dentre estes critérios se ressaltam os seguintes: pessoas com determinada idade; desenvolvimento psicossocial; categoria administrativa; classe social; modificações fisiológicas; grupo populacional. De maneira geral, a juventude situa-se como uma etapa transitória entre o período da infância e a maturidade (FRANCISCO; GROPPO, 2014). 
Para Mandelli; Soares; Lisboa (2011) a articulação entre a psicologia sócio histórica e a sociologia da juventude, torna possível a compreensão da juventude tal como um processo social em constituição, estreitamente relacionado com as características culturais e históricas, sem desprezar as características biológicas e geracionais. Utiliza-se a expressão juventudes para informar essas diferenças: se há juventudes, há também a necessidade de ações diferenciadas que sirvam de base para as diversidades.

A entrada na juventude requisita a saída do ambiente protegido da família, a discussão de valores, a introdução em novos grupos de convivência e o início de novos empreendimentos, com frequência, múltiplos e algumas vezes diferentes. Faz parte desse processo a ressignificação das dificuldades que afetam mais de perto os jovens, como também o reequacionamento de mecanismos e meios para enfrenta-los (GONÇALVES et al., 2008). Já a saída do individuo da juventude requer uma intervenção psíquica de elaboração de perdas e uma nova posição frente à vida. Torna-se importante relatar que um dos aspectos da juventude é o sentimento de inadequação no que se refere ao tempo, e por esse motivo, a necessidade de alterá-lo (MOREIRA; ROSÁRIO; SANTOS, 2011).

\section{Depressão}

De acordo com Cremasco; Baptista (2017) a depressão é o transtorno mental que mais acomete a população de modo geral, estima-se que em 2030 será a principal causa de incapacidade mental no mundo inteiro. De forma geral, esse transtorno é considerado como um grupo de sintomas fisiológicos e psicológicos, que podem se manifestar de maneira contínua ou em episódio, e sua intensidade apresenta variação de leve a severa.

Trata-se de uma patologia com sinais e sintomas específicos, e sua duração e gravidade são suficientes para ocasionar sérios comprometimentos sobre a capacidade de uma pessoa conduzir uma vida de normalmente. $O$ transtorno depressivo pode interferir de forma significativa na vida diária, nas interações sociais e no bem-estar geral do individuo (RIBEIRO, 2006).

Os principais sintomas apresentados na depressão são: humor deprimido durante quase todo o dia, diminuição acentuada do interesse ou prazer de realizar a maioria ou todas as 
atividades, significativo ganho ou perda de peso sem realizar dieta, aumento ou diminuição do apetite, inquietação ou retardo psicomotor, alterações do sono, cansaço ou perda de energia, diminuição da capacidade de pensar e concentrar-se, sentimentos de inutilidade, culpa exacerbada ou inadequada, confusão e pensamentos de morte recorrentes, ideação suicida sem a elaboração de um plano específico, tentativa de suicídio ou elaboração do plano específico para cometê-lo (CREMASCO; BAPTISTA, 2017).

Ainda de acordo com os autores supracitados, a depressão acomete mais a população feminina, sendo apontada na literatura como transtorno associado ao suicídio. Ressalta-se também que essa patologia gera sofrimento intenso e influencia de forma direta na qualidade de vida das pessoas, podendo causar tentativa de suicídio ou até mesmo o suicídio propriamente dito. Dessa forma, as estimativas apontam para a ocorrência de um aumento do número de suicídios para mais de 1,5 milhões de indivíduos anualmente, o que torna a temática um dos principais problemas de saúde pública no mundo.

Percebe-se uma estreita relação entre o transtorno depressivo e o suicídio, uma vez que o comportamento suicida pode ser concebido como um dos sintomas da depressão.

\section{Ideação Suicida}

A ideação suicida refere-se a pensamentos suicidas e ideias de autodestruição, incluindo atitudes, desejos e planos que o sujeito possui para pôr fim à sua própria vida. Não é anormal ter pensamentos e/ou ideais suicidas uma vez ou outra, já que estes pensamentos também integram o processo de desenvolvimento normal da transição da fase da infância para a juventude, na proporção que se enfrentam problemas existenciais e se está buscando uma compreensão acerca da vida, da morte e a significação da existência (MOREIRA; BASTOS, 2015).

Ainda conforme os autores citados anteriormente, os pensamentos suicidas são considerados anormais quando a execução destes se mostra ser a única resolução dos problemas para os jovens, tornando-se, portanto, um importante risco para a tentativa de suicídio ou suicídio. A intensidade com que esses pensamentos ocorrem, sua profundidade, como também o contexto em que emergem e a impossibilidade de libertar-se deles é que se 
constituem fatores que diferenciam um jovem saudável de um que está prestes a entrar em uma crise suicida. Nesse sentido, a ideação suicida pode ser percebida como um fator de risco para a efetivação do suicídio, juntamente com a desesperança e a depressão. Atitudes que demonstram arrogância e enfrentamento, que buscam expressar muita força interior, na realidade, podem tratar-se de um pedido de ajuda, de carinho, de limites, de manifestação de angústias e dúvidas.

O suicídio na população jovem no mundo inteiro aumenta cada vez mais. Sendo considerado como a terceira causa de morte na entre jovens. Este acontecimento é classificado em três condições: ideação suicida, tentativa de suicídio e a consumação do suicídio (BARBOSA et al., 2016). A ideação suicida precede o ato, sendo fundamental não somente a identificação precoce desses pensamentos, todavia, também, uma maior compreensão acerca das causas do seu aparecimento e os aspectos específicos dessa fase do desenvolvimento humano (MOREIRA; BASTOS, 2015).

\section{Suicídio}

A morte é um acontecimento temido pela maioria das pessoas; no entanto, pode ser percebida como um alívio para aqueles que não conseguem encontrar soluções para seus dilemas, e procuram, através de atitudes autodestrutivas, dar fim a própria vida. A juventude por ser uma etapa de desenvolvimento marcado por diferentes transformações biológicas, psicológicas e sociais que, comumente, são acompanhadas de angústias e conflitos, tem-se percebido, nos últimos anos, um aumento no comportamento suicida nessa população (MOREIRA; BASTOS, 2015).

É considerado suicídio todo caso de morte resultante direta ou indiretamente de um ato, negativo ou positivo, executado pela própria vítima, a qual já previa o resultado (MARBACK; PELISOLI, 2014).

O suicídio apresenta-se como um sério problema de saúde pública, e está entre as dez principais causas de óbito na população mundial de todas as idades. Além disso, o suicídio ocupa o terceiro lugar na população com idade entre 15 e 34 anos e o segundo lugar em indivíduos com mais de 65 anos, com taxas que variam de acordo com o contexto social, gênero, idade e meios utilizados. Aproximadamente metade de todas as mortes violentas 
acontecem em decorrência do suicídio, o que representa quase 1 milhão de vítimas por ano, e taxas que vão de 10 a 20 milhões de pessoas que tentam suicídio anualmente. Estima-se que em 2020, esse número de vítimas poderá chegar a 1,53 milhões, e de 10 a 20 vezes mais indivíduos realizarão tentativa de suicídio (SCHLÖSSER; ROSA; MORE, 2014).

Acredita-se que o suicídio é multifatorial, não podendo ser compreendido apenas por um ângulo e, na maior parte dos casos, há uma associação entre fatores de ordem psicológica, psiquiátrica, econômica, culturais, religiosa que precisam ser levadas em consideração (FUKUMITS; SCAVACINI, 2013).

Com relação aos fatores de risco relacionados ao suicídio, destacam-se os seguintes: tentativas prévias de suicídio, transtornos mentais (especialmente depressão e o uso abusivo e/ou dependência de álcool e outras drogas), histórico de suicídio na família, falta de apoio social, intenso pensamento suicida, acontecimentos estressantes e aspectos sociais e demográficos, como por exemplo, pobreza, baixo grau de escolaridade e desemprego. Embora não haja um conceito único aceitável, o suicídio envolve necessariamente um anseio consciente de morrer e a compreensão clara do que o a execução do ato pode ocasionar (MOREIRA; BASTOS, 2015).

Nesse sentido, é importante ressaltar que o suicídio se manifesta como consequência final de um conjunto maior de condições que colocam a vida em risco, considerando-as como atitudes suicidas (SCHLÖSSER; ROSA; MORE, 2014).

Faz-se necessário ressaltar, que, em se tratando de mulheres, a quantidade de casos é maior no que concerne a elaboração do plano e tentativa de suicídio, entretanto, o maior número de casos de suicídio é apresentado na população masculina, uma vez que os meios usados pelos homens são mais letais que os utilizados pelas mulheres (CREMASCO; BAPTISTA, 2017).

Acredita-se que, em muitas situações, o suicídio pode ser prevenido e que, em casos de crise, cujo risco para o suicídio é alto, o profissional deve tomar providências e cuidados efetivos na conduta clínica, ser receptivo, alerta e disponível a condição de conflito e manter contato com a família, médicos e/ou orientar a encaminhar para internação, caso seja necessário (FUKUMITSU; SCAVACINI, 2013). 


\section{Intervenção Clínica da Psicologia com o Jovem com Ideação Suicida}

O suicídio, como também os fatos que o antecedem, não é um acontecimento isolado, porém sua ocorrência se dá pelo sincronismo de diferentes fatores predisponentes que atravessam as diversas áreas da existência. Trata-se de um fato universal e complexo que afeta de forma completa as culturas, ideias e classes sociais, possuindo uma etiologia multifatorial, que envolve elementos de ordem biológica, genética, social, psicológica, cultural e ambiental que relacionam-se à vida pessoal e coletiva (BORBA; CUNHA, 2016).

A princípio, deve-se apontar que a prevenção e intervenção no suicídio não são possíveis de forma solitária e, dessa forma, o trabalho deve ser realizado em articulação com os familiares e outros profissionais envolvidos (FUKUMITSU, 2014).

No contexto da Psicologia clínica, o trabalho deve iniciar-se a partir da análise dos motivos antecedentes do suicídio procurando determinar sua relação com os estímulos internos que causaram o ato, na maioria das vezes circunstâncias de imenso sofrimento experimentadas anteriormente pelo individuo. Quando um fator atual aponta para a ocorrência de um sentimento assolador que é capaz de levar a um suicídio, é geralmente pelo motivo dele reeditar um acontecimento anterior de sofrimento, elaborando-o e tornando insuportável o momento atual. Nessas situações, o suicídio se manifesta como uma saída, uma forma possível de amenizar o sofrimento e a dor (CONSELHO FEDERAL DE PSICOLOGIA, 2013).

Neste sentido, o cuidado com as pessoas que pensam em suicidar-se é extremamente importante, uma vez que diariamente essa população aumenta. Apesar de ocasionar sérias consequências, grande parcela da população sabe pouco acerca de como lidar com uma pessoa que apresenta ideação suicida, e até mesmo como reconhecer as características desse tipo de comportamento. Tendo como resultado importantes dificuldades em agir com pessoas em situação de risco, inclusive por parte de diversos profissionais de saúde, que por não terem sido instruídos sobre como atuar nessa situação, acabam acreditando em mitos, como por exemplo, que os pacientes que falam em suicídio raramente vão o cometer; e que falar sobre o suicídio pode estimular atos suicidas (CERQUEIRA; LIMA, 2015).

Nesses casos, a atuação do psicólogo consiste especialmente em estabelecer uma comunicação mais pessoal com o sujeito em crise como também com seus familiares, 
averiguando a possível necessidade de ser realizada uma avaliação da situação por um psiquiatra (BORBA; CUNHA, 2016).

É necessário que o psicoterapeuta desenvolva empatia no que diz respeito ao sofrimento humano, ou seja, o profissional poderá se disponibilizar para uma aproximação do lugar onde o paciente se encontra e, através de sua disponibilidade interpessoal, a esperança de que o paciente possa reconhecer seus potenciais - com a finalidade de ampliar sua forma de enfrentamento de suas dificuldades - poderá ou não surgir. Dessa forma, um relacionamento terapêutico que preza pelo cuidado e não pela cura pode facilitar que o paciente ressignifique sua angústia existencial e encontre perspectivas de como lidar com seus conflitos. Entretanto, nem sempre tais características são bastante a ponto de garantir que o sujeito pare de acreditar que a morte seja mais agradável que a vida (FUKUMITSU, 2014).

Dentre as formas de intervenção, aponta-se o acompanhamento, que trata-se de uma experiência de atuação na área de saúde mental na qual está compreendida a observação, análise e tentativa de modificação da qualidade de vida de indivíduos acometidos por transtornos psiquiátricos. A finalidade maior é colaborar na organização e implementação do cuidado intensivo em saúde mental, com o objetivo de mudar uma vida paralisada que não tem mais capacidade de encontrar nenhuma saída, ou que apenas encontra como saída, a eliminação da própria vida (ESTELLITA-LINS; OLIVEIRA; COUTINHO, 2006).

A intervenção da psicologia é de grande importância e exerce papel primordial no cuidado às pessoas suicidas no qual a utilização da abordagem da Terapia Cognitivo Comportamental abrange os indivíduos com ideação suicida em que objetiva a uma reestruturação cognitiva associada à resolução de problemas. Essa abordagem compõe uma forma de atuação psicoterápica, a qual possibilita ao profissional terapeuta fazer a avaliação sobre qual a correlação entre as condutas que o paciente manifesta nos âmbitos sentimental e comportamental, através de uma situação de estímulo e, também qual o vínculo dessa correlação com o ambiente no qual essa pessoa encontra-se inserida (BORBA; CUNHA, 2016).

Ainda de acordo com os autores supracitados, um dos mais importantes aspectos dessa abordagem é o foco no problema; trata-se da utilização de uma série de procedimentos clínicos, cuja finalidade é principalmente a correção das perspectivas errôneas, as quais fundamentam comportamentos perturbados e impulsivos. 


\section{Orientações do Conselho Federal de Psicologia sobre o Suicídio}

É importante salientar que para se prevenir o suicídio ou para a promoção da vida não necessita entrar diretamente ou especificamente na discussão do suicídio, trata-se, principalmente, de valorizar ou promover entre as pessoas a temática da vida. Quantas vezes é imposto ao indivíduo que ela permaneça vivo, porém sem oferecer-lhe nenhuma condição de compreender por qual motivo ele está objetivando a morte, por que ele almeja essa morte. $\mathrm{O}$ sujeito simplesmente permanece suportando aquela situação por acreditar que é um pecado, por não querer gerar um sofrimento ainda maior para seus familiares, acaba enfrentando essa situação miserável de vida que lhe foi imposta, sem nem ao menos questioná-la, na maioria das vezes por não entendê-la, ou não acreditar saber que é possível transformá-la (CONSELHO FEDERAL DE PSICOLOGIA, 2013).

Dessa forma, a atuação de profissionais de saúde mental abrange imensa mobilização psíquica, já que o paciente os defronta com conflitos e ansiedade. Na situação de atendimento a pessoas com ideação ou tentativa de suicídio, essa movimentação conduz o profissional a entrar em comunicação com suas angústias, questionamentos, e dúvidas (ESTELLITA-LINS; OLIVEIRA; COUTINHO, 2006).

A intervenção psicológica a pessoas nessa situação, suscita questionamentos no que se refere a aspectos éticos, principalmente no que concerne à questão do sigilo. O exercício da profissão da Psicologia deve se embasar na ética para assegurar a interação adequada entre o profissional, cliente e sociedade, conforme valores importantes. Pode haver dificuldade para o Psicólogo realizar suas decisões e deliberações, uma vez que, atitudes humanas acontecem numa junção complexa de situações: experimentos podem ofender a dignidade do ser humano, um Psicólogo Clínico pode causar muita interferência na vida do paciente e o trabalho realizado em instituições pode causar conflito de interesses (ZANA; KOVÁCS, 2013).

O psicólogo deve ter consciência de que necessita ler mais, inteirar-se acerca do fenômeno, ciente que existem diferentes fatores de risco para o suicídio, como, por exemplo, um transtorno psiquiátrico, brigas no meio familiar e a perda de um emprego. Existe sempre uma vulnerabilidade psíquica que necessita ser entendida. Deve-se levar em consideração que 
é possível prevenir o comportamento suicida e, para que isso ocorra é necessário um efetivo planejamento e a criação de programas que envolvam diferentes profissionais capacitados para tal finalidade. A comunidade, obviamente, deve ser trabalhada simultaneamente. Não se pode trabalhar somente com os profissionais que atuam em postos de saúde, porém também com voluntários que desenvolvam algum trabalho em bairros, com as igrejas e também organizações não governamentais. A prevenção do comportamento suicida constitui-se em um importante desafio não somente para a Psicologia, como também para a sociedade em geral, por tratar-se de um desafio social, político e econômico (CONSELHO FEDERAL DE PSICOLOGIA, 2013).

\section{Considerações Finais}

A depressão é considerada como um grave problema de saúde pública a nível mundial, devido suas altas taxas de incidência. Sendo considerada também como o transtorno mental mais comum na população. A seriedade desse transtorno deve-se também ao fato de que ele é um importante fator de risco tanto para a ideação suicida como para o suicídio.

Dessa forma, este trabalho teve como objetivo identificar a relação entre depressão e ideação e descrever as intervenções clínicas da psicologia nesses casos.

A depressão na juventude, por ser um fato bastante comum, é preocupante, uma vez que a ocorrência desse transtorno eleva os riscos do aparecimento de pensamentos suicidas, tentativas de suicídio e até mesmo o suicídio propriamente dito.

É fundamental que os sinais e sintomas concernentes a depressão sejam detectados precocemente e que as medidas de tratamento e auxilio sejam tomadas em tempo hábil, com a finalidade de melhorar o quadro depressivo e prevenir a ideação suicida.

Para que ocorra a intervenção efetiva da Psicologia deve ser realizada a análise das causas que antecederam o comportamento suicida, para que se seja possível estabelecer sua associação com os estímulos internos que o causaram.

Dentre as formas de intervenção, destaca-se o acompanhamento, que é uma experiência de atuação na área de saúde mental que envolve a observação, análise e tentativa de modificação da qualidade de vida de pessoas com transtornos psiquiátricos.

Conclui-se, nesse sentido, que a depressão com ideação suicida na juventude é um problema que necessita de redobrada atenção, pela complexidade e seriedade que o 
caracteriza. Portanto, é importante a realização de mais estudos que abordem este tema e apresentem medidas que sirvam de prevenção para esses eventos.

\section{Referências}

ANDRADE, M. M.; et al. Adolescência, Escolha Profissional e Identificação:

Uma Revisão Sistemática. Id on Line Revista Multidisciplinar e de Psicologia. v. 10, n. 30, p. 178204. 2016. Disponível em: 〈https://idonline.emnuvens.com.br/id/article/view/459/613>. Acesso em: 30 de mar. 2017.

BARBOSA, A. K. L.; Bullying e sua relação com o suicídio na adolescência. Id on Line Revista Multidisciplinar e de Psicologia. v.10, n. 31, p. 202-220. 2016..

CREMASCO, G. S.; BAPTISTA, M. N. Depressão, motivos para viver e o significado do suicídio em graduandos do curso de psicologia. Estudos Interdisciplinares em Psicologia. v. 8, n.1, p. 22-37. 2017.

ESTEVES, F. C.; GALVAN, A. L. Depressão numa contextualização contemporânea. Aletheia. n. 24, p. 127-135. 2006. Disponível em: 〈http://pepsic.bvsalud.org/pdf/aletheia/n24/n24a12.pdf〉. Acesso em: 14 de fev. 2017.

FRANCISCO, J. C.; GROPPO, L. A. Adolescência(s) e juventude(s): considerações a partir de uma coexistência legal. Crítica Educativa. v. 2, n. 2, p. 275-294. 2016. Disponível em: $<$ http://www.criticaeducativa.ufscar.br/index.php/criticaeducativa/article/download/81/237>. Acesso em: 03 de maio 2017.

FUKUMITSU, K. O.; SCAVACINI, K. Suicídio e manejo psicoterapêutico em situações de crise: uma abordagem gestáltica. Revista da Abordagem Gestáltica. v. 19, n. 2, p. 198-204. 2013.

GONÇALVES, H. S.; et al. Problemas da juventude e seus enfrentamentos: um estudo de representações sociais. Psicologia \& Sociedade. V. 20, n. 2, p. 217-225. 2008.

LARANJEIRA, P. I. C. A relação entre depressão e ideação suicida em jovens adultos: o papel mediador da desesperança e da dor mental. 2015. 79f. Dissertação (Especialização em Psicologia Clínica e da Saúde) - Escola de Ciências Sociais, Universidade de Évora, Évora, 2015.

MANDELLI, M. T.; SOARES, D. H. P.; LISBOA, M. D. Juventude e projeto de vida: novas perspectivas em orientação profissional. Arquivos Brasileiros de Psicologia. v. 68, n. 2. 2011. Disponível em: <http://seer.psicologia.ufrj.br/index.php/abp/article/view/723/587>. Acesso em: 04 de maio 2017.

MARBACK, R. F.; PELISOLI, C. Terapia cognitivo-comportamental no manejo da desesperança e pensamentos suicidas. Revista Brasileira de Terapias Cognitivas. v. 10, n. 1, p. 122-129. 2014.

MOREIRA, L. C. O.; BASTOS, P. R. H. O. Prevalência e fatores associados à ideação suicida na adolescência: revisão de literatura. Revista Quadrimestral da Associação Brasileira de Psicologia Escolar e Educacional. V. 19, n. 3, p. 445-453. 2015. 
MOREIRA, J. O; ROSÁRIO, A. B. do.; SANTOS, A. P. do. Juventude e adolescência: considerações preliminares. PSICO. v. 42, n. 4, p. 457-464. 2011. Disponível em: $<$ http://revistaseletronicas.pucrs.br/ojs/index.php/revistapsico/article/viewFile/8943/7450>. Acesso em: 03 de maio 2017.

ORGANIZAÇÃO MUNDIAL DE SAÚDE. Depression and other common mental disorders: global health estimates. OMS, 2017. Disponível em: <http://apps.who.int/iris/bitstream/10665/254610/1/WHO-MSD-MER-2017.2-eng.pdf?ua=1>. Acesso em: 15 fev. 2017.

ORGANIZAÇÃO PAN-AMERICANA DA SAÚDE. Depressão. OPAS, 2016. Disponível em: $<$ http://www.paho.org/bra/index.php?option=com_content\&view=article\&id=5321:depressao-e-temade-campanha-da-oms-para-o-dia-mundial-da-saude-de-2017\&Itemid=839>. Acesso em: 15 fev. 2017.

RIBEIRO, P. C. P. Secretaria de Estado de Saúde. Atenção à Saúde do Adolescente. $1^{\text {a }}$ ed. Belo Horizonte: SAS/MG, 2006.

SCHLÖSSER, A.; ROSA, G. F. C.; MORE, C. L. O. O. Revisão: comportamento suicida ao longo do ciclo vital. Temas em Psicologia. v. 22, p. 133-145. 2014.

VASCONCELOS-RAPOSO, J.; et al. Níveis de ideação suicida em jovens adultos. Estudos de Psicologia. v. 33, n. 2, p. 345-354. 2016.

ZANA, A. R. O.; KOVÁCS, M. J. O Psicólogo e o atendimento a pacientes com ideação ou tentativa de suicídio. Estudos e Pesquisas em Psicologia. v. 13, n. 3, p. 897-921. 2013. Disponível em: <http://pepsic.bvsalud.org/scielo.php?script=sci_arttext\&pid=S1808-42812013000300006>. Acesso em: 15 de fev. 2017.

\section{Como citar este artigo (Formato ABNT):}

ALENCAR, Abigail Virgínia M. de; MARANHÃO, Thércia L. G.; CARVALHO, FERNANDES, Rivalina M.M.; RODRIGUES, Maria do Socorro. A Relação entre Depressão e Ideação Suicida na Juventude. Id on Line Revista Multidisciplinar e de Psicologia, 2018, vol.12, n.39, p.519-532. ISSN: 1981-1179.

Recebido: 09.01.2018

Aceito: 11.01 .2018 